\title{
Endosymbiont diversity among sibling weevil species competing for the same resource
}

\author{
Adrien Merville ${ }^{1,2^{*}}$, Samuel Venner ${ }^{1}$, Hélène Henri', Agnès Vallier ${ }^{2}$, Frédéric Menu' ${ }^{1}$, Fabrice Vavre ${ }^{1}$, \\ Abdelaziz Heddi $^{2}$ and Marie-Claude Bel-Venner ${ }^{1 *}$
}

\begin{abstract}
Background: Whereas the impact of endosymbionts on the ecology of their hosts is well known in some insect species, the question of whether host communities are influenced by endosymbionts remains largely unanswered. Notably, the coexistence of host species competing with each other, which is expected to be stabilized by their ecological differences, could be facilitated by differences in their endosymbionts. Yet, the composition of endosymbiotic communities housed by natural communities of competing host species is still almost unknown. In this study, we started filling this gap by describing and comparing the bacterial endosymbiotic communities of four sibling weevil species (Curculio spp.) that compete with each other to lay eggs into oak acorns (Quercus spp.) and exhibit marked ecological differences.
\end{abstract}

Results: All four species housed the primary endosymbiont Candidatus Curculioniphilus buchneri, yet each of these had a clearly distinct community of secondary endosymbionts, including Rickettsia, Spiroplasma, and two Wolbachia strains. Notably, three weevil species harbored their own predominant facultative endosymbiont and possessed the remaining symbionts at a residual infection level.

Conclusions: The four competing species clearly harbor distinct endosymbiotic communities. We discuss how such endosymbiotic communities could spread and keep distinct in the four insect species, and how these symbionts might affect the organization and species richness of host communities.

Keywords: Endosymbiosis, Host community, Curculio, Oak weevil, Infection pattern, Niche partitioning, Field study

\section{Background}

The last decade has seen a growing number of studies exploring microbial endosymbiosis among arthropods and its implication for the evolution of the host species. The impressive array and diversity of species harboring one or more type(s) of endosymbionts raises the possibility that such associations may play a predominant role in the ecology and evolution of both partners. Most studies that have considered symbiotic associations from the perspective of the host support the view that heritable symbionts offer their host an opportunity for rapid and deep evolutionary changes and for greater adaptation to a novel environment [1-3]. Symbionts are considered to be

\footnotetext{
*Correspondence: adrien.merville@univ-lyon1.fr; marie-claude.venner@univlyon $1 . \mathrm{fr}$

'Université de Lyon, Université Lyon 1, CNRS, UMR5558, Laboratoire de Biométrie et Biologie Evolutive, Villeurbanne, France

${ }^{2}$ INSA-Lyon, UMR203 BF2I, INRA, Biologie Fonctionnelle Insectes et Interactions, Bat. L. Pasteur 20 ave A. Einstein, Villeurbanne, France
}

a keystone in the diversification and expansion of the ecological niche of their host [3-5]. Thus, endosymbionts have been shown to assist their host in exploiting poor nutritional resources, notably by complementing its nutrition [6-8], often to the point that they become essential or obligatory symbionts for the host. From the differences observed between the endosymbiotic communities of various host populations, it has been suggested that symbionts may mediate the specialization of their hosts on distinct resources and thus promote the emergence of allopatric host races, which might subsequently lead to speciation [9-15].

Mutualistic facultative symbionts are also able to provide their host with other skills, such as broadening the range of temperatures they can tolerate [16], reinforcing their ability to resist natural enemies [17-20], or increasing their dispersal capacity [21-24]. These rapidly-acquired capacities are likely to promote the expansion of the host populations and the diversification of their habitat [1-5].

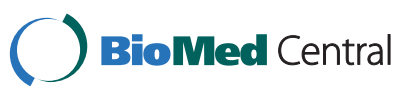


Besides these mutualistic relationships, facultative endosymbionts are also known to manipulate the reproduction of the host. In response to selective pressures favoring their vertical transmission, many symbionts, such as Wolbachia, have been shown either to skew the sex-ratio of the offspring toward females or to induce sterilization of uninfected females [25-27]. Such effects can affect both the population dynamics and gene flow, notably by promoting the reproductive isolation of infected hosts.

While firm knowledge is accumulating about the symbiont-mediated interactions between given host species and their environments, the possibility that endosymbionts may influence the organization of communities composed of several competing host species has so far been seldom investigated. The first empirical evidence of an endosymbiont-mediated relationship between competing host species has been recently provided from artificial protist communities kept under highly-controlled laboratory conditions. It was suggested that a green algal symbiont is essential for competing protist species to coexist, notably because the allelochemicals and the nutritional resource provided by this microorganism seem to balance the competitive capacity of the hosts [28]. Moreover, the issue of the competition between two hymenopteran parasitoid species has been shown experimentally to be tightly dependent on the presence of an inherited virus in one of the insect species [29]. These findings highlight the fact that the influence of microbial symbionts on the organization of competing host communities may have been considerably underestimated. The paucity of empirical data about the role of endosymbionts on competing host communities contrasts with the body of knowledge that has accumulated about extracellular symbiosis that has been shown to play a key role in the organization and composition of plant communities [30-32]. Microbial communities present in the soil often mediate nutrient uptake of plants, and might facilitate the coexistence of plant species in various ways, notably by allowing resource partitioning (reviewed in [33]).

A first support to the hypothesis that endosymbiosis does impact communities of insect species competing with each other would be that endosymbiotic profiles vary with respect to the host species. To study this aspect, we investigated endosymbiosis in natural oak weevil communities composed of four Curculio species - C. glandium (Marsham), C. elephas (Gyllenhal), C. pellitus (Boheman), and C. venosus (Gravenhorst) (Coleoptera, Curculionidae). These weevils coexist on the same individual oak trees (Quercus spp.) in southern Europe [34-36], where they compete for oak acorns that constitute the sole food resource for larval development and that have been shown to be highly limiting some years [37]. This biological system is relevant for examining our proposal in that $(i)$ the four oak weevil species are expected to host endosymbionts, like the few other Curculio species already described $[15,38]$ and (ii) they exhibit marked differences in their life history traits despite their recent divergence [36], suggesting that their coexistence is stabilized by means of ecological niche partitioning [37].

We provide an exhaustive description of the endosymbiotic meta-community harbored by the four oak weevil species in two distinct natural communities. Using a correlative and a quantitative approach, we found that endosymbiont communities differed across the four Curculio species, while being consistent within each species. We discuss the mechanisms possibly accounting for the contrasted infection patterns observed across these sibling species and the ways by which endosymbiosis may contribute to structuring communities of insect species competing with each other.

\section{Methods}

\section{Study system}

Four weevil species of the genus Curculio (C. glandium, C. elephas, C. pellitus and C. venosus) coexist on oak trees (Quercus spp.) in southern Europe [34]. In all four species, females lay eggs during summer within oak acorns, where larvae achieve their development before self-extracting and burrowing into the soil. Fully mature larvae then enter diapause during variable time periods -i.e., they spend from one to four years underground-, depending on the species or even on individuals within one species [37].

\section{Insect sampling}

Our study was conducted on two natural communities found in two sites in southern France (site 1: N45 35'; $\mathrm{E} 5^{\circ}$ 01'; site 2: $\mathrm{N}^{\prime} 5^{\circ} 45^{\prime} ; \mathrm{E}^{\circ}{ }^{16}$ ). Adult weevils were live-trapped on oak trees at each site weekly throughout one breeding season (June to September 2009) by beating branches with a wooden stick [39]. Insects were collected on a white sheet laid under the tree and their species was identified from morphological traits [34].

\section{DNA extraction}

Ovaries of 391 adult females caught on one of the two study sites and belonging to one of four weevil species (see Table 1) were dissected in buffer A (KCL $25 \mathrm{mM}$, $\mathrm{MgCl} 210 \mathrm{mM}$, Saccharose $250 \mathrm{mM}$, Tris-HCl $35 \mathrm{mM}$; $\mathrm{pH}=7.5)$ and homogenized in $500 \mu \mathrm{L}$ of buffer STE $(\mathrm{NaCl}$ $100 \mathrm{mM}$, Tris- $\mathrm{HCl} 10 \mathrm{mM}$ and EDTA $1 \mathrm{mM}, \mathrm{pH}=8$ ). These dissected tissue samples were individually subjected to DNA extraction by adding proteinase $\mathrm{K}(120 \mathrm{mg} / \mathrm{L})$ and SDS (sodium dodecyl sulfate: $0.5 \% \mathrm{~m} / \mathrm{v}$ ), and were then incubated for $2 \mathrm{~h}$ at $55^{\circ} \mathrm{C}$. RNA contamination was removed with the addition of RNaseA $(60 \mathrm{mg} / \mu \mathrm{L})$ for $1 \mathrm{~h}$ at $37^{\circ} \mathrm{C}$. DNA from each sample was then purified with phenol-chloroform extraction and precipitated using 
Table 1 Number of adult males and females of each weevil species collected at the two sites

\begin{tabular}{lccccc}
\hline Species & \multicolumn{2}{c}{ Females } & & \multicolumn{2}{c}{ Males } \\
\cline { 2 - 3 } \cline { 5 - 6 } & Site $\mathbf{1}$ & Site $\mathbf{2}$ & & Site $\mathbf{1}$ & Site 2 \\
\hline C. elephas & 74 & 38 & & 25 & 21 \\
C. glandium & 76 & 40 & & 26 & 29 \\
C. pellitus & 78 & 7 & & 36 & 7 \\
C. venosus & 64 & 14 & & 15 & 12 \\
\hline
\end{tabular}

isoamelic alcohol [40]. In addition, 171 adult males (Table 1) were automatically crushed individually with stainless steel beads shaken $20 \mathrm{~s}$ at $20 \mathrm{~Hz}$ (Tissue Lyser, Qiagen) [41] and their total DNA was extracted using NucleoSpin Tissue Kit (Macherey-Nagel).

\section{Characterization of the endosymbiotic lineages and phylogenetic analyses}

To get an overview of the endosymbionts harbored by the four Curculio species, bacterial $16 S$ rRNA amplification was performed individually on the ovaries of 95 females among which 40 C. elephas (24 from Site 1 and 16 from Site 2) and 20 females of each of the three remaining species (C. glandium, C. pellitus and C. venosus) comprising 10 females per site, except for $C$. pellitus from the site 2 for which only 5 females could be sampled. A $1.5 \mathrm{~kb}$ fragment was amplified using eubacterial universal primers (Table 2). Reactions were carried out in a $50 \mu \mathrm{L}$ final volume consisting of 2.5 units of Taq DNA polymerase (UptiTherm, Interchim, France), $2 \mathrm{mM} \mathrm{MgCl} 2,0.8 \mathrm{mM}$
dNTPs, $0.6 \mu \mathrm{M}$ primers, and 200 ng DNA template. The polymerase chain reaction (PCR) started at $95^{\circ} \mathrm{C}$ for $5 \mathrm{~min}$ and was followed by 26 cycles, each of these running $95^{\circ} \mathrm{C}$ for $30 \mathrm{~s}, 53^{\circ} \mathrm{C}$ for $1 \mathrm{~min}$ and $72^{\circ} \mathrm{C}$ for $2 \mathrm{~min}$; the final step of the reaction was $7 \mathrm{~min}$ at $72^{\circ} \mathrm{C}$. The PCR products were then pooled into eight groups, one per species and per site. Each of these pools was then subjected to cloning. In a first step, ten clones per pool were randomly selected and sequenced (Additional file 1; Beckman Coulter Genomics, Grenoble, France). Identifying these sequences revealed a predominance of three endosymbiont types (among which, mostly Curculioniphilus buchneri, and to a lesser extent, Wolbachia and Rickettsia; see below). Consequently, in a second step at least 130 clones were grown up for each pool (Additional file 1), and each clone was screened by diagnostic PCR using the specific primers corresponding to the three dominant bacterial types (Table 2). Clones that were grown successfully but that could be amplified by none of these three diagnostic PCRs (i.e., Curculioniphilus buchneri, Wolbachia and Rickettsia) were then sequenced (30 clones; see Additional file 1).

We characterized the bacteria hosted by Curculio females by performing phylogenetic analyses using the sequences obtained in this study together with the most similar ones found by Blast in Genbank. Multiple sequences were aligned using MUSCLE software [46]. The appropriate model of evolution was estimated with jmodeltest [47] for each set of sequences considered. Phylogenetic analyses were performed using maximum likelihood (ML) inference with Phyml v3.0.1 [48]. The

Table 2 Primers used for PCR diagnostic

\begin{tabular}{|c|c|c|c|c|c|c|}
\hline Target Species & Target gene & Primer name & Primer sequence $\left(5^{\prime}-3^{\prime}\right)$ & Product size (kb) & $\begin{array}{c}\text { Temp. } \\
\left({ }^{\circ} \mathrm{C}\right)\end{array}$ & Refs. $^{\dagger}$ \\
\hline Host & CytB rRNA & CBJ-10933 & TATGTACTACCATGAGGACAAATATC & 0.5 & 45 & {$[42]$} \\
\hline (Curculio sp.) & & CBN-11367 & ATTACACCTCCTAATTTATTAGGAAT & & & {$[42]$} \\
\hline \multirow[t]{2}{*}{ Eubacterial Universal Primers } & $16 \mathrm{~S}$ rRNA & 008For & AGA GTT TGA TCA TGG CTC AG & 1.5 & 53 & {$[43]$} \\
\hline & & 1487Rev & TAC CTT GTT ACG ACT TCA CC & & & $\left(*^{* *}\right)$ \\
\hline \multirow[t]{4}{*}{ Candidatus Curculioniphilus buchneri } & $16 \mathrm{~S}$ rRNA & $16 S-F$ & AGAGATCTGGAGGAATATCA & 0.4 & 52 & $(*)$ \\
\hline & & $16 S-R$ & CACTAAAGCATCTCTGCTAAAT & & & $(*)$ \\
\hline & GroEL & GroEL $2 \mathrm{~F}$ & ATG GGB GCT CAA ATG GTK AAA & 0.9 & 55 & {$[38]$} \\
\hline & & GroEL 2R & CTCTTCATTTCAACTTCNGTBGCA & & & {$[38]$} \\
\hline \multirow[t]{2}{*}{ Wolbachia } & $16 \mathrm{~S}$ rRNA & W-Spec F & CATACCTATTCGAAGGGATAG & 0.4 & 60 & [44] \\
\hline & & W-Spec R & AGCTTCGAGTGAAACCAATTC & & & [44] \\
\hline \multirow[t]{2}{*}{ Rickettsia } & $16 \mathrm{~S}$ rRNA & $\mathrm{RbF}$ & GCTCAGAACGAACGCTATC & 0.9 & 58 & [12] \\
\hline & & $\mathrm{RbR}$ & GAAGGAAAGCATCTCTGC & & & [12] \\
\hline \multirow[t]{2}{*}{ Spiroplasma } & ITS and $16 \mathrm{~S}$ rRNA & Spixo-16S F & TTAGGGGCTCAACCCCTAACC & 0.8 & 52 & [45] \\
\hline & & Spixo-16S R & TCTGGCATTGCCAACTCTC & & & {$[45]$} \\
\hline \multirow[t]{2}{*}{ Sodalis } & $16 \mathrm{~S}$ rRNA & Sodalis $370 \mathrm{~F}$ & CGRTRGCGTTAAYAGCGC & 0.2 & 55 & [38] \\
\hline & & 16SSod590R & AACAGACCGCCTGCGTACG & & & {$[38]$} \\
\hline
\end{tabular}

${ }^{*}$ Annealing temperature. ${ }^{\dagger}$ References: $\left({ }^{*}\right)$ this study; $\left.{ }^{* *}\right)$ nucleotides numbering from Escherichia coli, GenBank accession number J01859. 
models selected were GTR $+G$ for Rickettsia (16S, 873 sites) as well as for Spiroplasma (16S + ITS, 1405 sites), HKY85 +I for Serratia (16S, 503 sites), $K 80+I$ for Wolbachia (16S, 340 sites). The robustness of the nodes was assessed with 100 bootstrap replicates. Additionally, Bayesian phylogenies were performed with MrBayes v3.1.2 [49] using appropriate parameters leading to convergence between two runs. We then compared topologies obtained with the two methods using approximate unbiased (AU) [50] and Shimodaira and Hasegawa (SH) [51] tests available in the CONSEL program package [52]. No significant difference was detected between the two topologies for any of the bacteria.

To examine the possible co-diversification between Curculioniphilus buchneri, considered as the primary symbiont, and its hosts, we used the method previously described to build the phylogenies based on COI gene sequences retrieved from Genbank for the weevil phylogeny, and on two distinct markers, the $16 S$ rRNA sequence and the GroEL gene, for the bacterial phylogeny. Nine host-symbiont pairs were included in the analysis, among which the four Curculio species studied here, the hazelnut weevil C. nuсum that we collected in a French population near Lyon, and four Japanese Curculio species whose primary symbiont has already been described (C. cameliae, C. sikkimensis, C. robustus and C. dentipes; [38].). The models selected were TPM2 + I for C. buchneri (16S, 267 sites), GTR $+I+G$ for Curculioniphilus buchneri (GroEL gene, 915 sites) and for Curculio spp. (COI gene, 375 sites). For Curculioniphilus buchneri bacteria, the congruence of the 16S rRNA and the GroEL topologies was rejected by the reciprocal AU and SH tests (AU: $P=0.004$; SH: $P=0.014)$ probably because the $16 S$ topology was weakly resolved, which precluded the concatenation of both sequences. Then, we tested the global congruence between the symbiont and host phylogenies, using $16 S$ and GroEL gene for the bacterial tree separately, with the CopyCat program (The Cophylogenetic Analysis Tool, version 2.00.02 [53]). After checking the occurrence of global co-diversification, we further tested the association of each of the nine individual host-symbiont pairs using the $\mathrm{COI}$ and the GroEL phylogeny that provided the best resolved bacterial tree. Patristic distances were used as input matrix and the AxParafit program was run with 999 permutations [54].

\section{Diagnostic PCR}

After checking the quality of the DNA of each insect sample (by amplifying a $487 \mathrm{bp}$ fragment of the cytochrome $b$ gene of the insect; Table 2), 562 adult weevils (males or females from one of the four species; see Table 1) were screened for each of the five endosymbionts found in the first part of the study using their specific primers (i.e., $C$. buchneri, Wolbachia, Rickettsia, Spiroplasma and Sodalis; Table 2). Although diagnostic PCR might fail to detect an endosymbiont that might be too rare in the host individual, we considered in subsequent analyses that the insect was free of this bacterium. To check whether the successful PCRs were actually specific of the targeted symbiont, two DNA amplicons randomly selected per symbiont and per weevil species were sequenced. To discriminate between the two possible Wolbachia strains detected in our samples (see Results), we performed a Restriction Fragment Length Polymorphism following the PCR assay based on the $16 \mathrm{~s}$ rRNA sequence (PCR-RFLP) on each Wolbachia-infected weevil. For that purpose, $17 \mu \mathrm{l}$ of each PCR product was digested at $37^{\circ} \mathrm{C}$ for $3 \mathrm{~h}$ with $5 \mathrm{U}$ of AluI (Fermentas, Villebons/Y., France) and was separated on a $2 \%$ agarose gel electrophoresis during $1 \mathrm{~h}$ at $100 \mathrm{~V}$. One DNA sample from each Wolbachia strain and each host species was randomly selected and sequenced to check the accuracy of the bacterial lineage identified by PCR-RFLP.

\section{Data analysis}

In each weevil species, we used a generalized linear model (GLM) fitted with a binomial error and a logit-link function to test whether the probability for an insect to be infected by the predominant facultative endosymbiont depended on its sex, on the locality it came from, or both. The best-fitted model was selected for each endosymbiont according to the AIC criterion. Further, the effects of each variable selected in the model and the possible interactions between variables were tested by an analysis of deviance. All the analyses were performed with the $\mathrm{R}$ software v.2.12.0 (http://www.r-project.org/).

\section{Results}

\section{Endosymbiotic communities in the oak weevil communities}

On the basis of 1,358 $16 \mathrm{~S} r \mathrm{RNA}$ clones derived from the ovaries of females of the four oak weevil species collected at the two study sites, ten distinct bacterial lineages were identified (Additional file 1): Curculioniphilus buchneri, Wolbachia, Rickettsia, Spiroplasma, Sodalis, Serratia, Xenorhabdus, Brevundimonas, Erwinia and Escherichia. Xenorhabdus, Brevundimonas and Erwinia are known to be entomopathogenic and/or free-living bacteria. The identified strain of Escherichia corresponds to the vector used for the cloning step. The phylogenetic analysis of Serratia reveals that it is part of a monophyletic group of nonsymbiotic bacteria that are distant from those known as symbionts (data not shown). Furthermore, this bacterium was shown to infect very few insect individuals at any site and irrespective of the weevil species, suggesting that the Serratia strain identified in this study was likely to be an insect pathogen: we discarded it from further analysis. A single clone among 1,358 ones was identified as a Sodalis-like endosymbiont $(97,77 \%$ of similarity with the Sodalis strain found in C. sikkimensis). It 
was detected in the pool of C. elephas females sampled at the site 2, and could be a posteriori detected by means of individual PCR diagnostic in one of the 16 females of this pool. We could not detect this symbiont in any of the 562 oak weevils individually screened with the same method. Consequently, we conducted phylogenetic analyses on the five endosymbionts identified (i.e. Curculioniphilus, Wolbachia strains 1 and 2, Rickettsia, Spiroplasma) (Figures 1 and 2).

For the Curculioniphilus endosymbiont, four distinct sequences were obtained, one for each weevil species. We found statistical evidence of global co-diversification between weevil hosts and their primary symbiont on each dataset $(P=0.002$ for COI-GroEL comparison, $P=0.005$ for COI-16S comparison). In addition, the association of seven of the nine host-symbiont pairs individually tested was found significant (see Figure 1). The insignificant associations corresponded to Curculio elephas and C. dentipes with their symbionts, and could be explained by their weakly supported positions in the phylogenetic trees generated either by bootstrap or by Bayesian posterior probabilities. This finding suggests co-diversification between Curculioniphilus and its weevil hosts and implies that this endosymbiont has been acquired by a common ancestor of Curculio spp. and subsequently strictly vertically transmitted. The Wolbachia $16 s$ rRNA sequences revealed at least two distinct strains. The corresponding phylogeny (Figure 2A) indicates that one of them belongs to the Wolbachia supergroup A while the other one seems rather positioned at the edge of the Wolbachia supergroup $\mathrm{B}$, according to the way Wolbachia sequences are annotated in Genbank. While we detected Rickettsia in all four

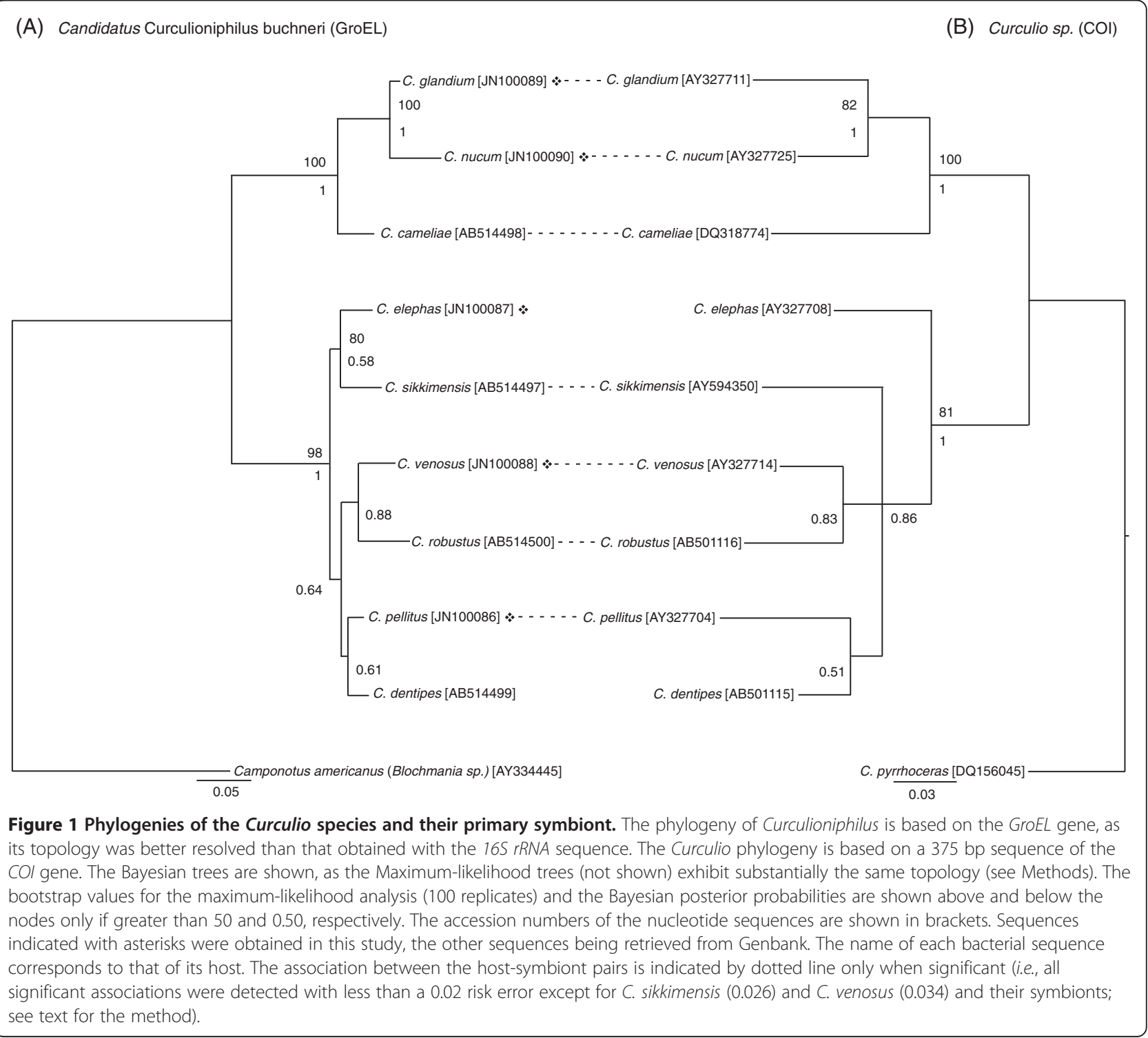



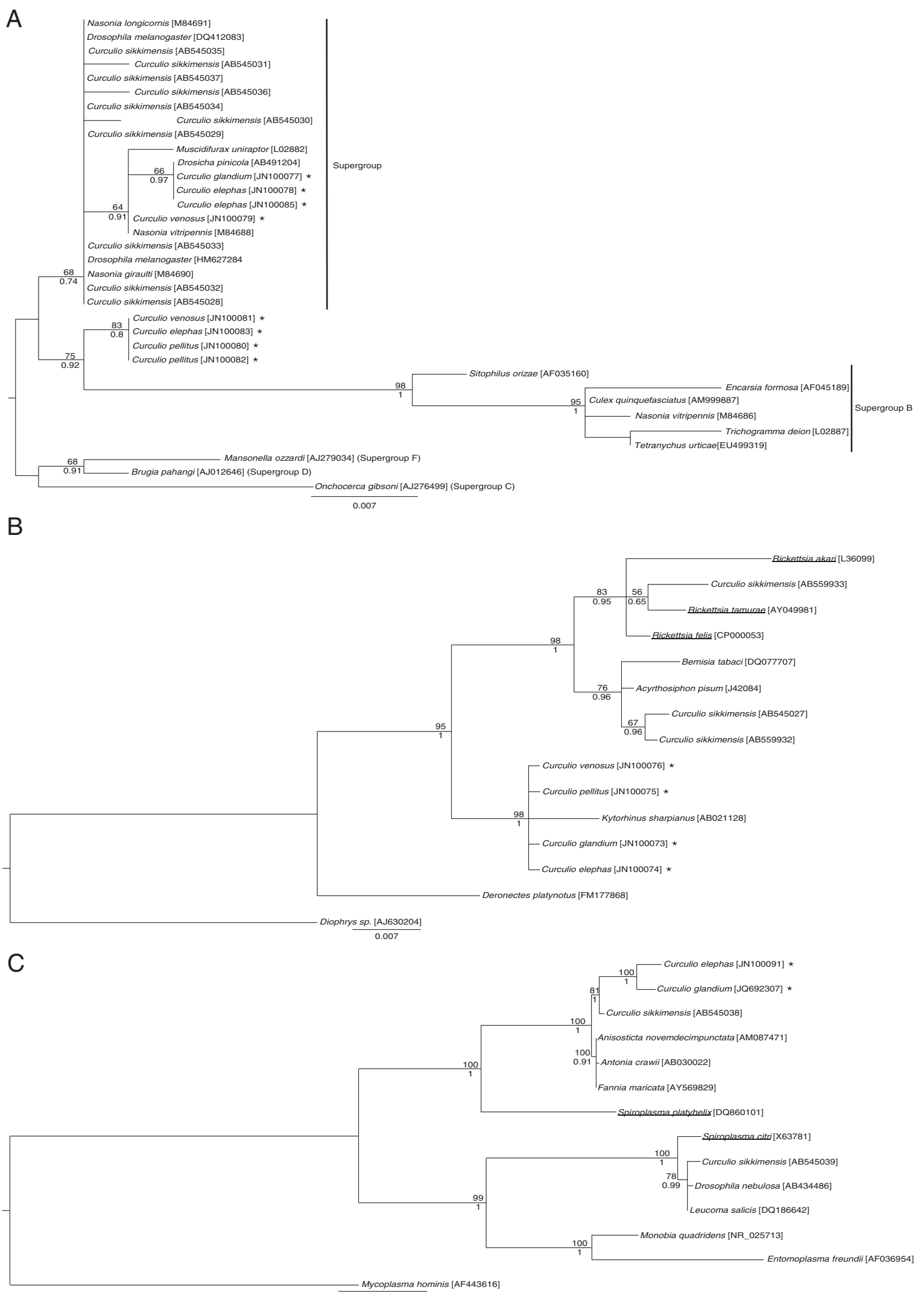

Figure 2 (See legend on next page.) 
(See figure on previous page.)

Figure 2 Phylogenies of the facultative endosymbionts identified in Curculio spp. (A) Wolbachia (340 bp unambiguously-aligned nucleotide sites, 165 rRNA sequences) is shown with indication of the supergroups A and B based on the annotation of sequences found in Genbank; (B) Rickettsia (873 sites, 165 rRNA sequences); (C) Spiroplasma (1405 sites, ITS and 16S rRNA sequences). The Bayesian trees are shown, as the Maximum-likelihood trees (not shown) exhibit substantially the same topology (see Methods). The bootstrap values for the maximum-likelihood analysis (100 replicates) and the Bayesian posterior probabilities are shown above and below the nodes only if greater than 50 and 0.50 , respectively. The accession numbers of the nucleotide sequences are shown in brackets. Sequences obtained from this study are indicated by asterisks. The name of each bacterial sequence corresponds to that of its host. Bacteria reported as non-endosymbiotic ones in the literature are underlined.

weevil species, we failed to discriminate them from one host species to the next. The phylogenetic analysis (Figure 2B) suggests that this bacterium is closely related to the Kytorhinus sharpianus endosymbiont (Coleoptera: Bruchidae, [55]). Finally, $3.33 \%$ and less than $1 \%$ of the bacterial clones derived from $C$. elephas and $C$. glandium females contained Spiroplasma, respectively: this bacterium was allied to the Curculio sikkimensis endosymbiont (Figure 2C, [15]).

Individual and multiple infection status of the oak weevils We have screened Curculioniphilus as well as the three other endosymbionts in each of 562 weevils collected (Table 1). Curculioniphilus is highly prevalent in adults from the four species irrespective of the sex or the study site (it was found in $90.5 \%$ C. elephas, $84.2 \%$ C. glandium, 98.4\% C. pellitus and $97.1 \%$ C. venosus; see Figure 3). The few uninfected individuals were essentially males (37 out of 44 uninfected individuals). Besides this so-called primary symbiont, three weevil species were shown to house their own predominant facultative symbiont (see Figure 3): 97.1\% of 171 C. glandium individuals were infected by the Wolbachia strain belonging to the supergroup A, $98.5 \%$ of 128 C. pellitus individuals were infected by the Wolbachia strain at the basis of the supergroup B, and 77.1\% of 105 C. venosus individuals hosted Rickettsia. In none of these species did the prevalence of the predominant secondary symbiont significantly vary according to either the sex or the study site (Table 3). A significant effect of the sex:locality interaction was statistically detected, however, on the prevalence of the supergroup A Wolbachia strain in C. glandium. This effect seems to be due to the unequal infection rate observed mostly in males between the two sites (infected males: $87 \%$ (site $1, \mathrm{n}=26$ ) vs 100\% (site 2, $\mathrm{n}$ = 29); infected females: $100 \%$ (site $1, \mathrm{n}=76$ ) vs $95 \%$ (site 2 , $\mathrm{n}=40$ ); Figure 3). This finding might stem from an unbalanced distribution of aged males in the two sites sampled (see discussion), and seems to be of low relevance owing to the small sample size of males as compared with that of females. In the two oak weevil communities studied, C. elephas differed from the three other species in that it lacked a predominant symbiont and exhibited Spiroplasma in addition to the three other secondary symbionts, all four endosymbionts being detected at a residual frequency. The three other weevil species, in addition to their predominant secondary symbiont, also harbored the other endosymbionts -except Spiroplasmaat a residual frequency. Considering the individual host level, multiple infections commonly occurred in the four species, mostly due to the co-occurrence of the primary symbiont and the predominant secondary symbiont (Figure 3). Interestingly, the two strains of Wolbachia were never detected simultaneously in any individual despite their marginal co-occurrence within the same species and study site.

\section{Discussion}

This study thoroughly describes the endosymbiotic communities hosted simultaneously by four weevil sibling species that coexist, compete with each other for a limiting resource (i.e., oak acorns as egg-laying sites) $[37,56,57]$ and display contrasted ecological traits $[37,58]$. We found that while all four species hosted the same primary endosymbiont, they harbored markedly distinct communities of secondary endosymbionts. Even if our results are strictly correlative, since we did not investigate the actual role of each symbiont on its host, this finding is compatible with our proposal that stable coexistence of host species competing with each other, which is expected to be ensured by their ecological differences, might be mediated by their endosymbionts.

Curculioniphilus buchneri was identified in all four weevil species using an a priori-free method based on $16 \mathrm{~S}$ $r R N A$ cloning and sequencing. This symbiont is known to be part of a distinct clade of Gammaproteobacteria, and is hosted by as many as 9 Curculio species. These insects feed on achenes or capsules and are specialist of perennial plants, including Theaceae (Camellia spp.) and Fagaceae (Corylus, Castanea.and Quercus spp.) [36,38]. The congruence observed between the phylogenies of $C$. buchneri and that of their host (Figure 1) suggests that this symbiont has been ancestrally acquired [4], which sustains its primary status. C. buchneri was found at a very high infection rate in the weevils screened in this study, and the few insects that did not carry C. buchneri were almost exclusively males (Figure 3 ). As primary endosymbionts are most often involved in complementing the host's diet, this finding suggests that this bacterium might have 


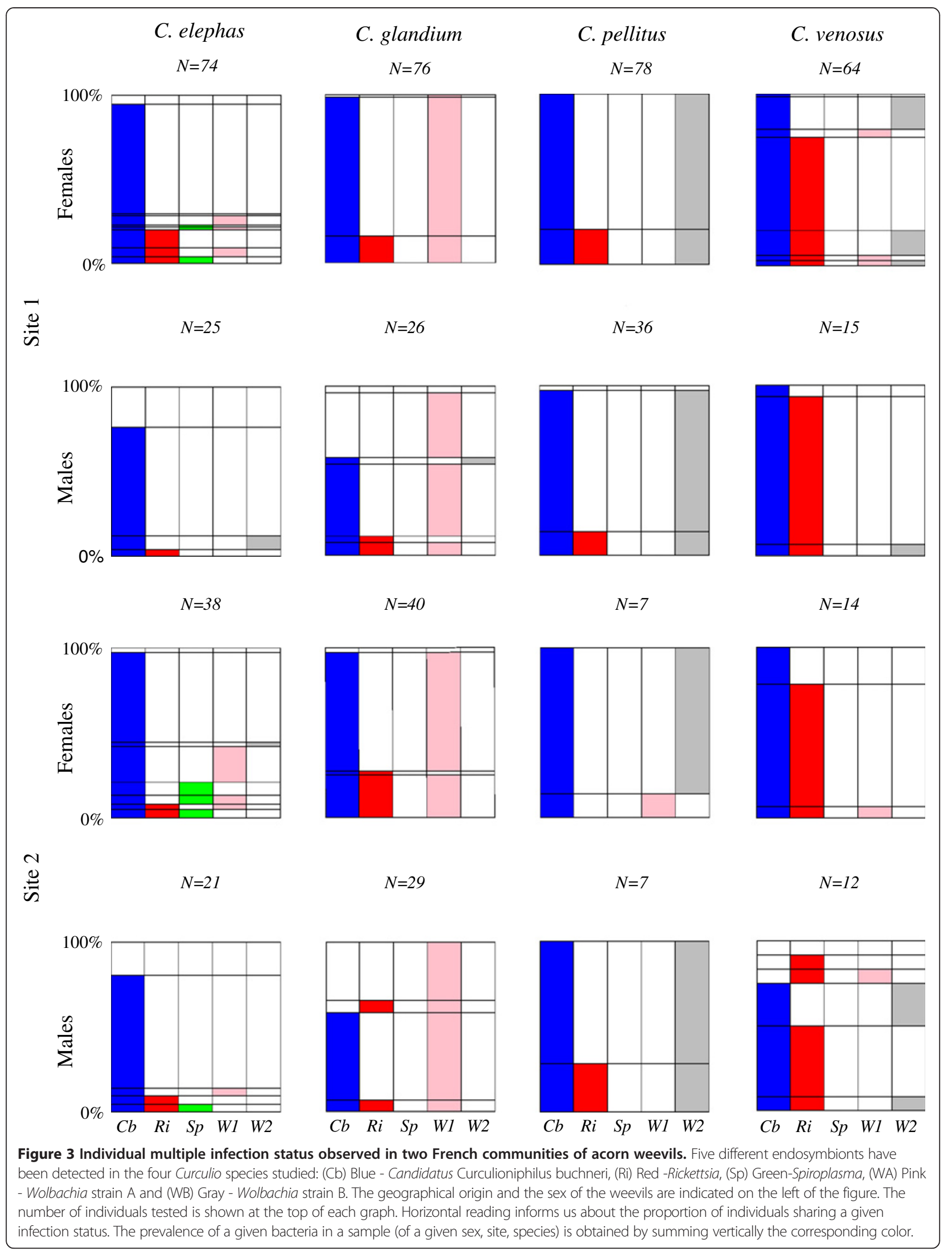


Table 3 Probability for a predominant facultative symbiont to infect an individual host as a function of its sex and of the study site

\begin{tabular}{llcc}
\hline Variable & Deviance & df & P \\
\hline Rickettsia (C. venosus) & & & \\
Site & 0.31589 & 1 & 0.57409 \\
Sex & 0.66578 & 1 & 0.41453 \\
Site:Sex & 2.75129 & 1 & 0.09718 \\
Wolbachia A (C. glandium) & & & \\
Site & 0.0003 & 1 & 0.98704 \\
Sex & 1.746 & 1 & 0.18635 \\
Site:Sex & 8.9503 & 1 & $\mathbf{0 . 0 0 2 7 7}$ \\
Wolbachia B (C.pellitus) & & & \\
Site & 1.9356 & 1 & 0.16415 \\
Sex & 0.0747 & 1 & 0.78467 \\
Site:Sex & 3.7132 & 1 & 0.05398 \\
\hline
\end{tabular}

Each analysis was performed on the weevil species shown in brackets. For each bacterium, the best-fitted generalized linear model (logistic regression) is shown, and was selected on the basis of the Akaike Information Criterion (AIC =117.2 for Rickettsia, 42.48 for Wolbachia A and 22.88 for Wolbachia B). The P-values for respective explanatory variables were obtained with chi-square statistics.

been lost during adulthood by a few males and hence, that it might be essential to the larval rather than the adult stage [59].

Three of the secondary symbionts (two distinct clades of Wolbachia and one Rickettsia) were commonly detected in all four species, whereas the fourth one, Spiroplasma, was found exclusively in C. elephas. This pattern resembles that of an endosymbiotic metacommunity, in which the endosymbiotic communities of each host population/species comprise all the bacterial types and are connected to one another by means of horizontal transfers [2,4,60-65]. Horizontal transfers have been reported to occur between parasitoid species sharing the same host [65], and are likely to occur between the four weevil species under study here, because of the concomitant presence of larvae from several species occasionally feeding on the same individual acorns (unpublished results). Evidence of such transfers remains to be established, however, notably through the analysis of more variable molecular sequences of the symbionts.

While at first sight the set of secondary endosymbionts detected was found to be identical across the four weevil species, a finer-tuned, quantitative analysis revealed that these ones had clearly distinct endosymbiotic communities. The four insect species differed from one another in that, with the exception of C. elephas, each had its own major secondary symbiont. Two questions remain to be addressed: (i) why not all symbionts have spread to all four weevil species, and (ii) why different symbionts predominate in the different host species? Multiple infections are likely to be counter-selected since at the individual host level, competition between endosymbionts for the resource, might lead to competitive exclusion and/or to increased cost for the host $[66,67]$. Although we observed the presence of a particular endosymbiont correlating with a weevil species, we cannot infer at this time its actual impact on host phenotype. However, assuming that symbionts have spread in response to selective pressures, two alternatives can be envisaged, depending on whether each of the major secondary symbionts engages in a mutualist interaction with its host or manipulates its reproduction $[2,4]$.

First, mutualistic endosymbionts provide their hosts with higher benefits than costs and are expected to invade the population rapidly $[5,20]$. The net benefit for a host to house a given endosymbiont might greatly depend on its physiological or ecological requirements. These needs might differ from one host species to the other, notably when these ones interact in different ways with the same environment $[11,64]$. In the oak weevil communities, the marked ecological differences observed between the four species $[37,58]$ would coincide with unequal probability for a given symbiont to spread. Conversely, we cannot rule out that the marked ecological differences between the four weevil sibling species might be due to the major symbionts having a distinct impact on their host. Hence, the different symbionts might facilitate the partitioning of ecological niches between their hosts by providing them with expanded or new skills, thereby possibly leading to unequal nutritive assistance [6,7,68-71], dispersal capacity [21-24], or ability to resist natural enemies among host species $[17-20,72]$.

Second, the probability for reproductive parasites to successfully invade their host heavily relies on the efficiency of their transmission from mother to offspring. In each of the four weevil species, males and females were frequently and equally infected, which makes male-killing or feminization processes unlikely. For instance, the infection patterns observed would rather be compatible with cytoplasmic incompatibility (CI) [25-27]. Notably, the two strains of Wolbachia identified on the basis of $16 s r R N A$, that are found to be the major secondary symbionts of $C$. pellitus and C. glandium, show almost complete mutual exclusion at the individual host level (Figure 3), suggesting mutual bidirectional CI. Should this mechanism actually occur, these Wolbachia strains would have been prevented from invading $C$. elephas and $C$. venosus populations because of their too low infection rate (reviewed in [73]). Interestingly, endosymbiont-mediated $\mathrm{CI}$ has theoretically been shown to facilitate and reinforce reproductive isolation and speciation [25], and such endosymbiont-mediated species enrichment would therefore be expected particularly in communities composed of sibling host species. However, empirical evidence for this mechanism is still scarce and much debated [73-77]. 


\section{Conclusions}

Our findings raise the question of whether intracellular micro-organisms might contribute to the structure of natural communities of host species competing with each other, either by accelerating their ecological divergence or by reinforcing their reproductive isolation, possibly leading to speciation. Exploring this proposal calls for both theoretical development and empirical investigations describing the endosymbiotic communities housed by various arthropod communities composed of sibling and competing host species. It should then be possible to test the predictions that communities harboring diverse secondary symbionts have greater species richness and include more closely related species than those deprived of symbionts. It would be worth running experiments in parallel to elucidate the actual impact endosymbionts have on their host, which still remains largely unknown in most natural systems.

\section{Data accessibility}

DNA sequences have been deposited at Genbank with the accession numbers AB514497-AB514504, AB507714AB507716, and JN100059-JN10090. Phylogenetic trees have been deposited at TreeBase (http://purl.org/phylo/treebase/ phylows/study/TB2:S13815 ).

\section{Additional file}

Additional file 1: Identification of the clones sequenced in the four weevil species. To identify the endosymbiotic diversity, clones were sequenced in adult females belonging to the four weevil species and live-trapped in two sites (see Methods). We described the best hit blast of each clone and the ratio of the number of similar nucleotides to the total number of nucleotides (called Identities).

\section{Competing interests}

The authors declare that they have no competing interests.

\section{Authors' contributions}

This study is part of the PhD thesis of AM who is focused on the implication of endosymbionts on the structure of sibling insect communities. MCBV and $\mathrm{AH}$ supervised this PhD at the interface between community ecology and endosymbiosis interactions. MCBV and SV suggested the exploration of endosymbiotic communities within competing species, supervised and contributed to the ecological research. FM provided his experience about weevil ecology and contributed to the experimental scheduling. $\mathrm{AH}$ and FV gave their expertise in the interactions existing between insects and their bacterial endosymbionts. AV conducted the fine dissections of the female ovaries and molecular analyses from DNA purification, cloning, up to sequencing. $\mathrm{HH}$ contributed to the methodological setting up of diagnostic PCRs and supervised the phylogenetic analyses. All authors read and approved the final manuscript.

\section{Authors' information}

MCBV, SV and FM are focused on evolutionary and community ecology. FV $\mathrm{AH}, \mathrm{HH}$ and $\mathrm{AV}$ have interest in associations between insects and bacteria endosymbionts. This work is part of the PhD of AM who develops an integrative research project linking ecological and symbiotic aspects.

\section{Acknowledgements}

We thank P.F. Pélisson, B. Rey and F. Débias for their help on insect community surveys, and S. Balmand, J. Blugeot and C. Vincent-Monégat for their contribution to the molecular and phylogenetic analyses. We are also grateful to D. Charif for her statistical advices and we thank two anonymous reviewers that helped us improving an earlier draft of the manuscript. This work was funded by the French National Research Agency (ANR: CoCoReCo project JC09-470585), by the CNRS, and by Envirhonalp ('Sante' et Environnement' Environment Cluster).

Received: 3 September 2012 Accepted: 30 January 2013

Published: 4 February 2013

References

1. Feldhaar $H$, Gross $\mathrm{R}$ : Immune reactions of insects on bacterial pathogens and mutualists. Microbes Infect 2008, 10:1082-1088.

2. Clark EL, Karley AJ, Hubbard SF: Insect endosymbionts: manipulators of insect herbivore trophic interactions? Protoplasma 2010, 244:25-51.

3. Ferrari J, Vavre F: Bacterial symbionts in insects or the story of communities affecting communities. Phil Trans Biol Sci 2011, 366:1389-1400.

4. Moran NA, McCutcheon JP, Nakabachi A: Genomics and evolution of heritable bacterial symbionts. Annu Rev Genet 2008, 42:165-190.

5. Himler AG, Adachi-Hagimori T, Bergen JE, Kozuch A, Kelly SE, Tabashnik BE, Chiel E, Duckworth VE, Dennehy TJ, Zchori-Fein E, Hunter MS: Rapid spread of a bacterial symbiont in an invasive whitefly is driven by fitness benefits and female bias. Science 2011, 332:254-256.

6. Douglas AE: Nutritional interactions in insect-microbial symbioses: aphids and their symbiotic bacteria Buchnera. Annu Rev Entomol 1998, 43:17-37.

7. McCutcheon JP, McDonald BR, Moran NA: Convergent evolution of metabolic roles in bacterial co-symbionts of insects. Proc Natl Acad Sci U S A 2009, 106:15394-15399.

8. Lamelas A, Gosalbes MJ, Moya A, Latorre A: New clues about the evolutionary history of metabolic losses in bacterial endosymbionts, provided by the genome of buchnera aphidicola from the aphid cinara tujafilina. Appl Environ Microbiol 2011, 77:4446-4454.

9. Leonardo TE, Muiru GT: Facultative symbionts are associated with host plant specialization in pea aphid populations. Proc R Soc B 2003, 270:S209-S212.

10. Simon J-C, Carré S, Boutin M, Prunier-Leterme N, Sabater-Mun B, Latorre A, Bournoville R: Host-based divergence in populations of the pea aphid: insights from nuclear markers and the prevalence of facultative symbionts. Proc R Soc B 2003, 270:1703-1712.

11. Tsuchida $T$, Koga R, Fukatsu T: Host plant specialization governed by facultative symbiont. Science 1989, 2004:303.

12. Gottlieb Y, Ghanim M, Chiel E, Gerling D, Portnoy V, Steinberg S, Tzuri G, Horowitz AR, Belausov E, Mozes-daube N, Kontsedalov S, Gershon M, Gal S, Katzir N, Zchori-fein E: Identification and localization of a rickettsia sp. In bemisia tabaci (homoptera: aleyrodidae). Appl Environ Microbiol 2006 72:3646-3652.

13. Chiel E, Gottlieb Y, Zchori-Fein E, Mozes-Daube N, Katzir N, Inbar M, Ghanim $\mathrm{M}$ : Biotype-dependent secondary symbiont communities in sympatric populations of bemisia tabaci. Bull Entomol Res 2007, 97:407-413.

14. Gueguen G, Vavre F, Gnankine O, Peterschmitt M, Charif D, Chiel E, Gottlieb Y, Ghanim M, Zchori-Fein E, Fleury F: Endosymbiont metacommunities, mtDNA diversity and the evolution of the bemisia tabaci (hemiptera: aleyrodidae) species complex. Mol Ecol 2010, 19:4365-4378.

15. Toju H, Fukatsu T: Diversity and infection prevalence of endosymbionts in natural populations of the chestnut weevil: relevance of local climate and host plants. Mol Ecol 2010, 20:853-868

16. Montllor CB, Maxmen A, Purcell AH: Facultative bacterial endosymbionts benefit pea aphids acyrthosiphon pisum under heat stress. Ecol Entomol 2002, 27:189-195.

17. Oliver KM, Russell JA, Moran NA, Hunter MS: Facultative bacterial symbionts in aphids confer resistance to parasitic wasps. Proc Natl Acad Sci U S A 2003, 100:1803-1807.

18. Haine ER, Moret $Y$, Siva-jothy MT, Rolff J: Antimicrobial defense and persistent infection in insects. Science 2008, 322:1257-1259.

19. Brownlie JC, Johnson KN: Symbiont-mediated protection in insect hosts. Trends Microbiol 2009, 17:348-354. 
20. Jaenike J, Unckless R, Cockburn SN, Boelio LM, Perlman SJ: Adaptation via symbiosis: recent spread of a Drosophila defensive symbiont. Science 2010, 329:212-215

21. Grenier A-M, Nardon C, Nardon P: The role of symbiotes in flight activity of Sitophilus weevils. Entomol Exp Appl 1994, 70:201-208.

22. Heddi A, Grenier AM, Khatchadourian C, Charles H, Nardon P: Four intracellular genomes direct weevil biology: nuclear, mitochondrial, principal endosymbiont, and Wolbachia. Proc Natl Acad Sci U S A 1999, 96:6814-6819.

23. Leonardo TE, Mondor EB: Symbiont modifies host life-history traits that affect gene flow. Proc R Soc B 2006, 273:1079-1084.

24. Goodacre SL, Martin OY, Bonte D, Hutchings L, Woolley C, Ibrahim K Thomas CFG, Hewitt GM: Microbial modification of host long-distance dispersal capacity. BMC Biol 2009, 7:32.

25. Bordenstein SR: Symbiosis and the origin of species. In Insect Symbiosis. Edited by Bourtzis K, Miller TA. Boca Raton, FL: CRC Press; 2003:283-304.

26. Werren JH, Baldo L, Clark ME: Wolbachia: master manipulators of invertebrate biology. Nat Rev Microbiol 2008, 6:741-751.

27. Merçot H, Poinsot D: Infection by Wolbachia: from passengers to residents. C R Biol 2009, 332:284-297.

28. Müller JP, Hauzy C, Hulot FD: Ingredients for protist coexistence: competition, endosymbiosis and a pinch of biochemical interactions. J Anim Ecol 2011, 81:222-232.

29. Patot S, Allemand R, Fleury F, Varaldi J: An inherited virus influences the coexistence of parasitoid species through behaviour manipulation. Ecol Lett 2012, 15:603-610.

30. Harnett DC, Wilson GWT: Mycorrhizae influence plant community structure and diversity in tallgrass prairie. Ecology 1999, 80:1187-1195.

31. Reynolds HL, Packer A, Bever JD, Clay K: Grassroots ecology: plantmicrobe-soil interactions as drivers of plant community structure and dynamics. Ecology 2003, 84:2281-2291.

32. Van der Heijden MGA, Bardgett RD, Straalen MV: The unseen majority: soil microbes as drivers of plant diversity and productivity in terrestrial ecosystems. Ecol Lett 2006, 11:296-310.

33. Bever JD, Dickie IA, Facelli E, Facelli JM, Klironomos J, Moora M, Rillig MC, Stock WD, Tibbett M, Zobel M: Rooting theories of plant community ecology in microbial interactions. Trends Ecol Evol 2010, 25:468-478.

34. Hoffmann A: Faune de France Coléoptères Curculionides (Deuxième partie). Paris, France: Fédération Française des Sociétés de Sciences Naturelles; 1954.

35. Coutin R: Original characteristics of the evolving cycles of some European weevil species: Curculio elephas Gyll., C. nucum L., C. glandium Marsh., C. venosus Grav. and C. villosus F. Memoires de la Societe royale belge d'Entomologie 1992, 35:259-266.

36. Hughes J, Vogler AP: The phylogeny of acorn weevils (genus curculio) from mitochondrial and nuclear DNA sequences: the problem of incomplete data. Mol Phylogenet Evol 2004, 32:601-615.

37. Venner S, Pélisson PF, Bel-Venner MC, Débias F, Rajon E, Menu F: Coexistence of insect species competing for a pulsed resource: toward a unified theory of biodiversity in fluctuating environments. Public Library of Science One 2011, 6:e18039.

38. Toju H, Hosokawa T, Koga R, Nikoh N, Meng XY, Kimura N, Fukatsu T: "Candidatus curculioniphilus buchneri," a novel clade of bacterial endocellular symbionts from weevils of the genus curculio. Appl Environ Microbiol 2010, 76:275-282

39. Schauff ME: Collecting and preserving insects and mites: tools and techniques. Washington: USDA Misc Publ n¹443: Museum of Natural History; 1986.

40. Sambrook J, Russell DW: Molecular cloning: a laboratory manual. 3rd edition. New York, NY: Cold Spring Harbor Laboratory Press; 2001

41. Pélisson PF, Henri H, Bel-Venner MC, Allemand R, Merville A, Menu F, Venner S: Identification at the larval stage of four curculio species coexisting on oak trees using PCR-RFLP. Entomol Exp App/ 2011, 138:77-82.

42. Simon C, Frati F, Beckenbach A, Crespi B, Liu H, Flook P: Evolution, weighting, and phylogenetic utility of mitochondrial gene sequences and a compilation of conserved polymeras chain reaction primers. Ann Entomol Soc Am 1994, 87:651-701.

43. Lefèvre C, Charles H, Vallier A, Delobel B, Farrell B, Heddi A: Endosymbiont phylogenesis in the dryophthoridae weevils: evidence for bacterial replacement. Mol Biol Evol 2004, 21:965-973.

44. Werren $\mathrm{JH}$, Windsor DM: Wolbachia infection frequencies in insects: evidence of a global equilibrium? Proc R Soc B 2000, 267:1277-1285
45. Duron O: Never completely trust a model: insights from cytoplasmic incompatibility beyond wolbachia-drosophila interactions. Heredity 2008, 101:473-474.

46. Edgar RC: MUSCLE: a multiple sequence alignment method with reduced time and space complexity. BMC Bioinforma 2004, 5:1-19.

47. Posada D: jModelTest: phylogenetic model averaging. Mol Biol Evol 2008, 25:1253-1256.

48. Guindon S, Gascuel O: A simple, fast, and accurate algorithm to estimate large phylogenies by maximum likelihood. Syst Biol 2003, 52:696-704.

49. Ronquist F, Huelsenbeck JP: MrBayes 3: Bayesian phylogenetic inference under mixed models. Bioinformatics 2003, 19:1572-1574.

50. Shimodaira $H_{1}$ Hasegawa M: Multiple comparisons of loglikelihoods with applications to phylogenetic inference. Mol Biol Evol 1999, 16:1114-1116.

51. Shimodaira $\mathrm{H}$ : An approximately unbiased test of phylogenetic tree selection. Syst Biol 2002, 51:492-508.

52. Shimodaira $H$, Hasegawa M: CONSEL: for assessing the confidence of phylogenetic tree selection. Bioinformatics 2001, 17:1246-1247.

53. Meier-Kolthoff JP, Auch AF, Huson DH, Göker M: COPYCAT: cophylogenetic analysis tool. Bioinformatics 2007, 7:898-900

54. Legendre $P$, Desdevises $Y$, Bazin E: A statistical test for host-parasite coevolution. Syst Biol 2002, 51:217-234.

55. Fukatsu T, Shimada M: Molecular characterization of rickettsia sp in a bruchid beetle kytorhinus sharpianus. Appl Entomol Zool 1999, 34:391-397.

56. Sork VL, Bramble J, Sexton O: Ecology of mast-fruiting in three species of North American deciduous oaks. Ecology 1993, 74:528-541.

57. Leibold MA, McPeek MA: Coexistence of the niche and neutral perspectives in community ecology. Ecology 2006, 87:1399-1410.

58. Pélisson PF, Bel-Venner MC, Rey B, Burgevin L, Martineau F, Fourel F, Lecuyer C, Menu F, Venner S: Contrasted breeding strategies in four sympatric sibling insect species: when a proovigenic and capital breeder copes with a stochastic environment. Funct Ecol 2012, 26:198-206.

59. Mansour K: On the so-called symbiotic relationship between coleopterous insect and intracellular microorganisms. Q J Microsc Sci 1934, 77:255-272.

60. Boyle L, O'Neill SL, Robertson HM, Karr TL: Interspecific and intraspecific horizontal transfer of wolbachia in drosophila. Science 1993, 260:1796-1799.

61. Sandström JP, Russell JA, White JP, Moran NA: Independent origins and horizontal transfer of bacterial symbionts of aphids. Mol Ecol 2001, 10:217-228

62. Russell JA, Latorre A, Sabater-Muñoz B, Moya A, Moran NA: Side-stepping secondary symbionts: widespread horizontal transfer across and beyond the Aphidoidea. Mol Ecol 2003, 12:1061-1075.

63. Russell JA, Moran NA: Horizontal transfer of bacterial symbionts: heritability and fitness effects in a novel aphid host. Society 2005 71:7987-7994.

64. Tsuchida T, Koga R, Horikawa M, Tsunoda T, Maoka T, Matsumoto S, Simon JC, Fukatsu T: Symbiotic bacterium modifies aphid body color. Science 2010, 330:1102-1104.

65. Huigens ME, de Almeida RP, Boons PAH, Luck RF, Stouthamer R: Natural interspecific and intraspecific horizontal transfer of parthenogenesisinducing wolbachia in trichogramma wasps. Proc Roy Soc Lond B Biol Sci 2004, 271:509-515.

66. Mouton L, Dedeine F, Henri H, Boulétreau M, Profizi N, Vavre F: Virulence, multiple infections and regulation of symbiotic population in the wolbachia-asobara tabida symbiosis. Genetics 2004, 168:181-189.

67. Oliver KM, Moran NA, Hunter MS: Costs and benefits of a superinfection of facultative symbionts in aphids. Proc $R$ SOC B 2006, 273:1273-1280.

68. Dowd PF: Insect fungal symbionts: a promising source of detoxifying enzymes. J Ind Microbiol Biotechnol 1992, 9:149-161.

69. Tokuda $\mathrm{G}$, Watanabe $\mathrm{H}$ : Hidden cellulases in termites: revision of an old hypothesis. Biol Lett 2007, 3:336-339.

70. Brownlie JC, Cass BN, Riegler M, Witsenburg JJ, Iturbe-Ormaetxe I, McGraw EA, O'Neill SL: Evidence for metabolic provisioning by a common invertebrate endosymbiont, wolbachia pipientis, during periods of nutritional stress. PLOS Pathog 2009, 5:e1000368.

71. Carpenter KJ, Horak A, Keeling PJ: Phylogenetic position and morphology of spirotrichosomidae (parabasalia): new evidence from leptospironympha of cryptocercus punctulatus. Protist 2010, 161:122-132.

72. Oliver KM, Moran NA, Hunter MS: Variation in resistance to parasitism in aphids is due to symbionts not host genotype. Proc Natl Acad Sci U S A 2005, 102:12795-12800. 
73. Engelstädter J, Telschow A: Cytoplasmic incompatibility and host population structure. Heredity 2009, 103:196-207.

74. Shoemaker DD, Katju V, Jaenike J: Wolbachia and the evolution of reproductive isolation between drosophila recens and drosophila subquinaria. Evolution 1999, 53:1157-1164.

75. Marshall JL: The allonemobius-wolbachia host-endosymbiont system: evidence for rapid speciation and against reproductive isolation driven by cytoplasmic incompatibility. Evolution 2004, 58:2409-2425.

76. Telschow A, Hammerstein P, Werren JH: The effect of wolbachia versus genetic incompatibilities on reinforcement and speciation. Evolution 2005, 59:1607-1619.

77. Jaenike J, Dyer KA, Cornish C, Minhas MS: Asymmetrical reinforcement and wolbachia infection in drosophila. PLoS Pathog 2006, 4:e325.

doi:10.1186/1471-2148-13-28

Cite this article as: Merville et al:: Endosymbiont diversity among sibling weevil species competing for the same resource. BMC Evolutionary Biology 2013 13:28.

\section{Submit your next manuscript to BioMed Central and take full advantage of:}

- Convenient online submission

- Thorough peer review

- No space constraints or color figure charges

- Immediate publication on acceptance

- Inclusion in PubMed, CAS, Scopus and Google Scholar

- Research which is freely available for redistribution 